\title{
Use of the Ilizarov apparatus to improve alignment in proximal humeral fractures treated initially by a unilateral external fixator
}

\author{
Shabir Ahmed Dhar · Mohammed Farooq Butt • \\ Mohammed Ramzan Mir · Murtaza Fazal Ali · \\ A. A. Kawoosa
}

Received: 8 August 2008/Accepted: 29 October 2008/Published online: 28 November 2008

(c) Springer-Verlag 2008

\begin{abstract}
One of the recommended methods for the management of displaced unstable proximal humeral fractures is the unilateral external fixator. In polytrauma cases this method may be especially useful for the stabilisation of these fractures. However, problems may arise if the fracture is fixed in a malaligned or distracted position. Conversion of the treatment concept to other modalities is difficult because of the problem of pin tract infection. The Ilizarov apparatus provides an useful method in such situations, as it allows distraction, translation and compression without the need for further anaesthesia and additional fixation.
\end{abstract}

Keywords Proximal humerus · Malaligned ·

Ilizarov fixator

\section{Introduction}

Most proximal humeral fractures are not sufficiently displaced or angulated to require surgical management. It is estimated that $20 \%$ of all proximal humeral fractures should be treated surgically [1]. Many techniques of fixation have been described in the literature with treatment options focused on the displaced fracture fragments because these may have limited vascularity and may benefit from reduction and fixation [2].

Amongst the prominent indications for the stabilisation of fracture of the neck of the humerus is a displaced unstable fracture in a patient with multiple trauma. The

S. A. Dhar $(\bowtie) \cdot$ M. F. Butt $\cdot$ M. R. Mir .

M. F. Ali - A. A. Kawoosa

The Government Hospital for Bone and Joint Surgery,

Bagat Barzulla, Srinagar 190005, Kashmir, India

e-mail: shabirdhar@yahoo.co.in goal of treatment in such cases is to stabilise the bone allowing an early range of motion.

We report a series of three polytrauma cases where due to unacceptable position of the fracture fragments within the unilateral external fixator, an Ilizarov fixator was added in each case to the preexisting half pins and fracture reduction and union achieved by sequential distraction, translation and compression.

\section{Materials and methods}

From January 2005 to April 2008 three cases of polytrauma with an injury severity score $>18$ were admitted to the Government hospital for bone and joint surgery with one of the injuries being a displaced fracture of the surgical neck of the humerus. The fracture was stabilised with external fixation applied with two half pins proximal as well as distal to the fracture. The patients were stabilised in terms of measurable parameters over a period of 2 weeks during which their coexisting injuries were managed. Once the patients were stabilised and $\mathrm{pO}_{2} / \mathrm{FiO}_{2}$ normalised, the patients were shifted back to the orthopaedic unit. In all these cases the reduction in terms of radiologic parameters was unacceptable. Due to high possibility of the pin tract infection, the operative concept of open reduction and internal fixation was precluded (Figs. 1, 2).

In all three cases the neutralising bar was removed and the half pins affixed to an Ilizarov external fixator. No anaesthesia was required. Depending on fracture configuration and displacement, the fracture area was distracted initially then translated and finally compressed. This was done using hinges which allowed translation and angulation in the direction required. The threaded rods allowed distraction as well as compression to be applied. The 


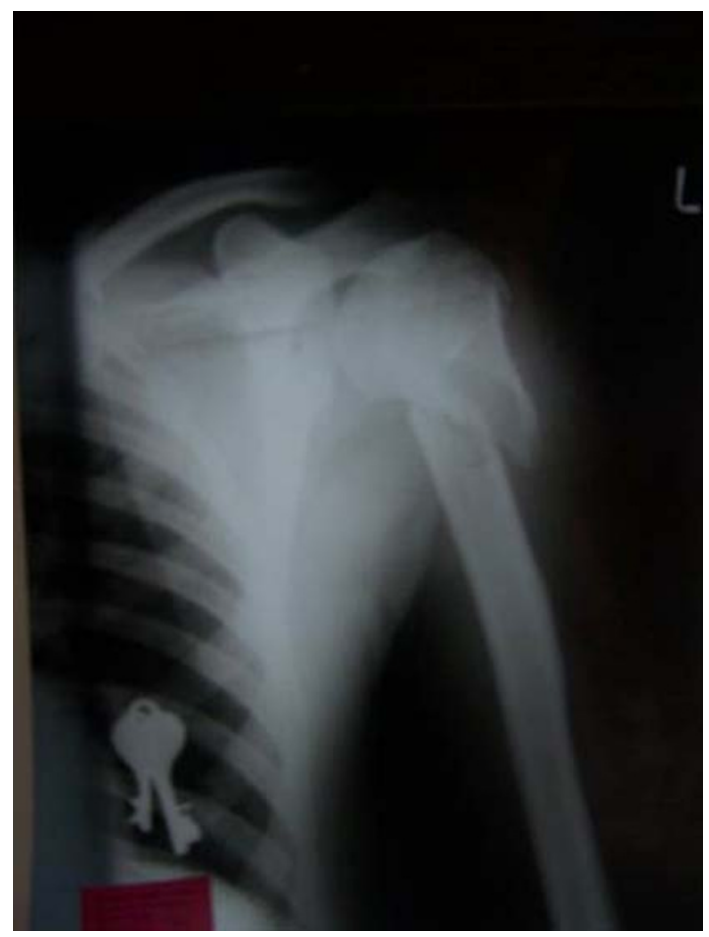

Fig. 1 Radiograph showing the fracture of the proximal humerus

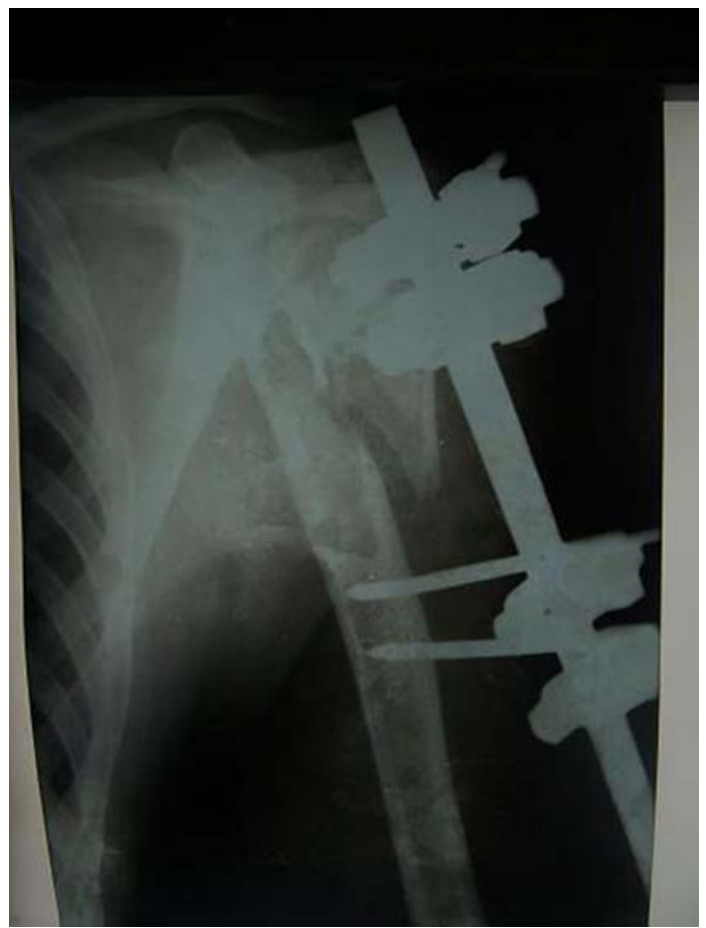

Fig. 2 Post-fixation radiograph showing malalignment

compression within the fixator was maintained for 8 weeks.

During this period the patient underwent physiotherapy to maintain range of motion. At 8 weeks radiologic and clinical stress testing was used to assess union. The fixator

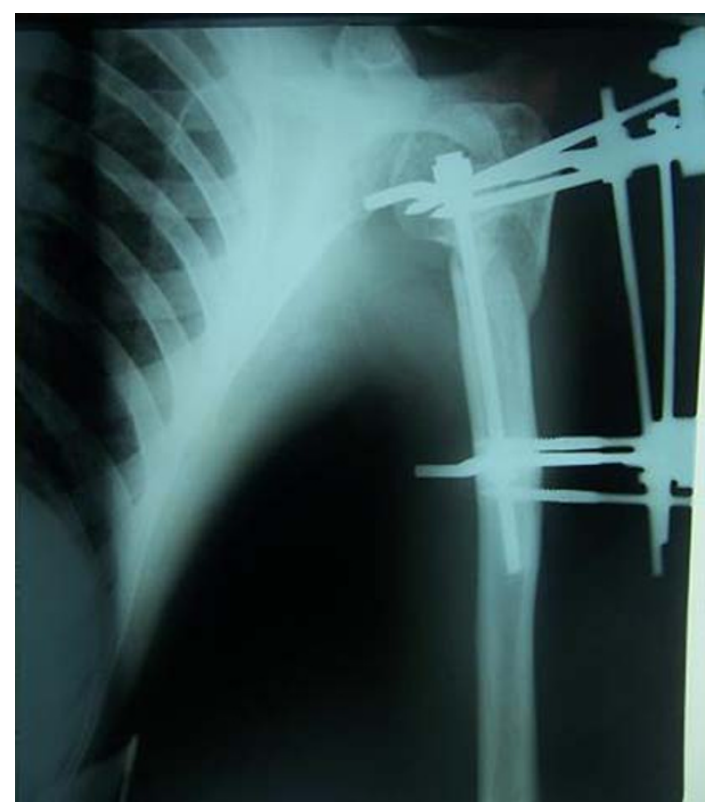

Fig. 3 Improvement of the alignment with the use of an ilizarov external fixator

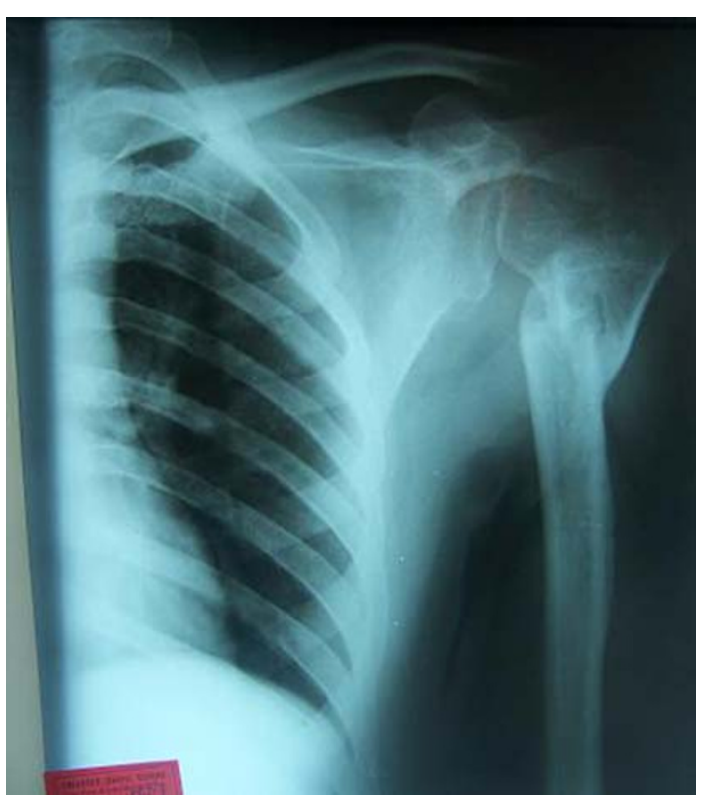

Fig. 4 Showing union in an acceptable position

was removed and patient allowed restricted activity for 1 month. After 1 month the patient was allowed to indulge in normal activities (Figs. 3, 4).

\section{Results}

All three patients showed union at 8 weeks. At a final follow-up of (4 months, 7 months, and 3 years, 7 months) 
all patients showed satisfactory union with a score of $>80$ as per the criteria established by Neer et al. [1].

\section{Discussion}

Surgical neck fractures of humerus are often under treated and are associated with a relatively high risk of malunion or non-union [3]. The goal of reduction and fixation of a proximal humeral fracture is to obtain nearly anatomic reduction and stable fixation to allow an early range of motion [4]. In polytrauma cases it is often difficult to manage such fractures properly at the outset as damage control orthopaedics is the preferred modality of treatment in such situations [5].

Using external fixator in such a situation to stabilise the proximal humerus is a viable option. Placement of two half pins proximal and distal to the fracture connected by a neutralising bar prevents further blood loss and can even be the final treatment modality [6]. However, at times, the use of external fixator is associated with the occurrence of pin tract infection. The conventional external fixator can also, at times, cause distraction and the inability to move the fragments onto each other with necessary force whilst the over distraction is maintained [7].

A combination of these two factors made open reduction difficult, whilst retaining the requirement for conversion osteosynthesis [8].

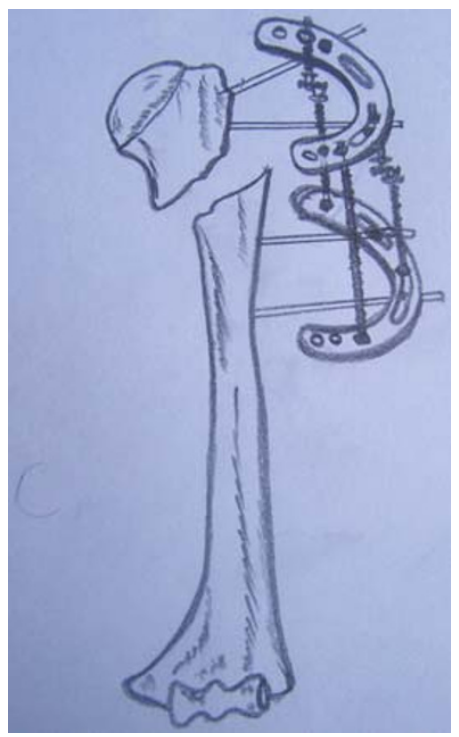

Fig. 5 Two arches were used for greater versatility. Hinges connecting the arches allowed translation and correction of angulation. The threaded rods were used to achieve distraction and final compression

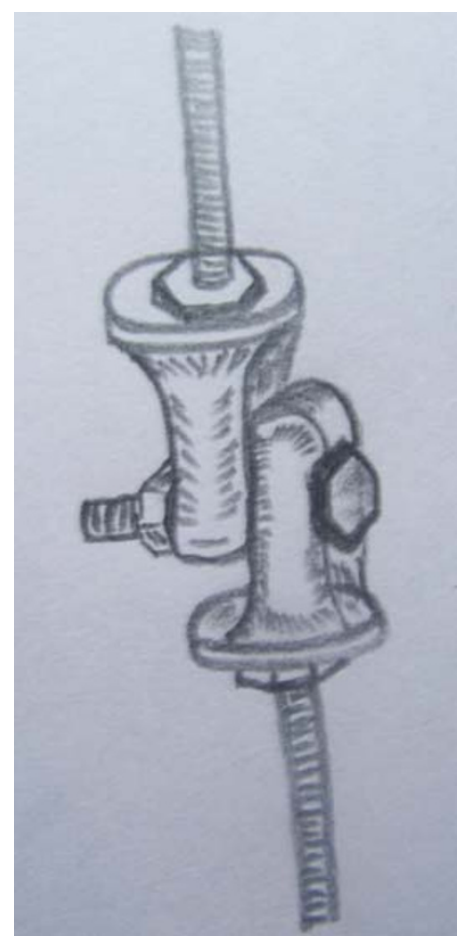

Fig. 6 This hinge assembly allows correction of angulation. Translation is achieved if additional hexagonal nuts are used

The Ilizarov fixator provides the orthopaedic surgeon with the possibility of distraction, compression and translation [9].

We utilised the two pins already placed on either side of the fracture. By sequentially applying distraction, translation and compression we were able to reduce the distraction in all our cases and improve alignment to acceptable limits. Due to the possibility of applying compression, we were able to facilitate union further. Both patients returned to their previous profession which incidentally was carpentry. Neither reported any sequel of pin tract problems at final follow up (Fig. 5, 6).

We feel that using the Ilizarov method in the conversion mode or perhaps primarily provides the following advantages:

1. Prevention of further devitalisation of bony fragments.

2. Reduction of infective complications.

3. Adjustability.

4. Early range of motion and rehabilitation.

\section{References}

1. Neer CS (1970) Displaced proximal humeral fractures II: treatment of 3 and 4 part displacement. J Bone Joint Surg 52-A:1090-1103

2. Vallier HA (2007) Treatment of proximal humerus fractures. J Orthop Trauma 21:7 
3. Sarmiento A, Waddell JP, Latta LL (2002) In diaphysial humeral fractures: treatment options Trauma. Instr Course Lect 51:257-269

4. Mourdian WH (1988) Displaced proximal humeral fractures: Seven years experience with a modified Zickel supracondylar device. Clin Orthop 212:209-218

5. Dhar SA, Mir MR et al (2008) Damage control orthopaedics' in patients with delayed referral to a tertiary care center; experience from place where composite trauma center do not exist. JTMC 2:2

6. Kristiansen B, Kofoed H (1987) External fixation of displaced fractures of the proximal humerus: technique and preliminary results. JBJS 69-B:643
7. Hammer R, Helland $P$ (1987) Improved skeletal fixation for unstable fractures. Clin Orthop 217:230-242

8. Dhar SA, Butt MF, Hussain A et al (2008) Management of lower limb fractures in poly trauma patients with delayed referral in a mass disaster. The role of the Ilizarov method in conversion osteosynthesis. Injury 39:947-951

9. Dhar SA, Mir MR, Ahmed MS et al (2008) Acute peg in hole docking in the management of infected non union of long bones. Int Orthop 32:559-566 